\title{
Determinación de la efectividad de una composición etnofarmacológica a base de seis extractos de plantas medicinales para el tratamiento de la hipertensión arterial
}

\section{Determination of the effectiveness of a composition etnofarmacológica with six extracts of medicinal plants for the treatment of the arterial hypertension}

\section{Carlos Ariel Rentería Jiménez* \\ RESUMEN}

El tratamiento de enfermedades como la hipertensión hace parte del conocimiento médico tradicional de las comunidades negras e indigenas de la región del Chocó biogeográfico colombiano. Con el objetivo de validar la efectividad de una composición etnofarmacológica a base de extractos acuosos de seis plantas medicinales, se realizaron análisis fitoquímicos para determinar su composición y se aplicaron en pacientes con esta patología para observar los efectos. Los resultados mostraron una presunción muy positiva para la presencia de compuestos lactónicos como cardiotónicos o terpenlactonas, y altamente positiva de compuestos fenólicos y leucoantocianidinas, que tienen una reconocida actividad biológica antioxidante e hipotensora. De la aplicación de entrevistas se obtuvo que $98.3 \%$ de los pacientes sometidos al tratamiento etnofamacológico expresaron sentir enorme mejoría en estado de ánimo, eliminando agotamiento, cansancio, fatiga y debilidad, inclusive desde la primera semana de iniciar el tratamiento. Se concluyó que es posible que los metabolitos secundarios presentes en la composición etnofarmacológica contribuyan al tratamiento de la hipertensión.

Palabras clave: Composición etnofarmacológica; Hipertensión; Plantas medicinales.

\begin{abstract}
The treatment of diseases as the hypertension is part of the traditional medical knowledge of the black and indigenous communities of the Choco bioegraphic region of Colombia. Because of validating the effectiveness of a etnofarmacology composition with watery extracts of six medicinal plants, phytochemistry analyses were realised to determine their composition and they were applied in patients with this pathology to observe the effects. The results showed a presumption very positive for the presence of compound lactónicos as cardiotónicos or terpenlactonas, and highly positive of phenolic compounds and leucoantocianidinas, which have one recognized biological activity tension antioxidant and. From the application of interviews it was obtained that $98.3 \%$ of the patients submissive the etnofarmacology treatment would express to feel enormous improvements in mood, being eliminated exhaustion, fatigue, fatigue and weakness, including from the first week initiating the treatment. It concluded that it is possible that the present secondary metabolites in the etnofarmacology composition contribute to the treatment of the hypertension.
\end{abstract}

Keywords: Etnofarmacology composition; Hypertension; Medicinal plants.
* Investigador de proyectos especiales, Instituto de Investigaciones Ambientales del Pacífico (IIAP), Quibdó, Colombia. e-mail: carielrenteria@hotmail.com

Recibido: 24 de septiembre, 2010 Aceptado: 14 de octubre de 2010 


\section{INTRODUCCIÓN}

El hombre ha encontrado en las plantas satisfacción a sus necesidades fundamentales (alimento, abrigo, casa, fabricación de utensilios, etc.), pero también ha descubierto que son capaces de aliviar sus dolencias y en algunos casos, curar enfermedades. Esto ha ocurrido desde tiempos inmemoriales y numerosas culturas han dejado testimonio de ello al trasmitirlos de forma oral, de generación en generación. La estrategia fundamental de investigación del IIAP, se orienta a lograr un salto en la calidad de vida de los pueblos del Chocó biogeográfico colombiano. Para lograr esto, se hace necesario investigar y defender la riqueza del conocimiento médico tradicional de las comunidades negras e indígenas de la región, que encuentra una de sus múltiples manifestaciones en el tratamiento que médicos tradicionales hacen a pacientes que sufren enfermedades de alta ocurrencia, como es el caso de la hipertensión arterial.

La hipertensión arterial (HTA), entendida como enfermedad crónica asintomática que se caracteriza por una elevación de presión arterial sistólica(PAS) mayor o igual a $120 \mathrm{~mm} \mathrm{Hg}$ y una presión arterial diastólica(PAD) mayor o igual a $80 \mathrm{~mm}$ Hg [Joint National Comitteon Prevention, Detection, and Treatment of High Blood Pressure (JNC-VII) 2003], se considera hoy como una de las principales problemáticas de salud pública. En la actualidad 7.,1 millones de personas mueren como resultado de tensión arterial elevada y se reconoce como una de las tres primeras causas de morbi-mortalidad mundial y nacional, uno de los primeros lugares de causas de incapacidad, uno de los principales motivos de consulta médica y una enfermedad con serias repercusiones a nivel económico y social (Bacon et al. 2004; Barrera et al. 2000; Blumenthal et al. 2002; Kaplan 2000, citado por: Fernández et al. (2003); JNC-VII 2003; OMS 2005; Organización Panamericana de la Salud (OPS) 2003; Sheu et al. 2003; Velasco y Hernández 2001; Whelton et al. 2002).

En Colombia, se estima que más de 10 millones de personas presentan HTA (22.8\% de la población adulta), siendo la región pacífica colombiana la que presenta la prevalencia de hipertensión más elevada (28\%) (Rodríguez et al. 2007).

En este artículo se presentan los resultados obtenidos en el municipio de Quibdó y sus alrededores, en relación con la comprobación de la efectividad de una composición etnofarmacológica a base de extracto acuoso de seis plantas medicinales para el tratamiento de la hipertensión arterial, propuesta por el médico tradicional Manuel Arcindo García Martínez para tratar la hipertensión.

El objetivo de este artículo es validar la efectividad de una composición etnofarmacológica a base de extractos acuosos de seis plantas medicinales para el tratamiento de la hipertensión arterial.

\section{ASPECTOS METODOLÓGICOS}

El desarrollo del estudio comprendió varias fases interrelacionadas; se inició con la identificación y descripción de las plantas medicinales utilizadas en la preparación de la composición etnofarmacológica y se determinó el contenido químico de la misma, así como la efectividad del tratamiento médico tradicional aplicado a la cura y/o alivio de pacientes hipertensos.

Identificación y descripción de las plantas medicinales. Se llevó a cabo esta etapa con el propósito fundamental de describir e identificar taxonómicamente las diferentes especies de plantas medicinales utilizadas para la preparación de la composición etnofarmacológica para el tratamiento de la hipertensión arterial. Para este aspecto en particular y entendiendo el proceso progresivo en el que se desarrolló este estudio, se tomó el registro de las plantas utilizadas, a fin de verificar su identificación taxonómica, completar las descripciones botánicas y hacer una revisión bibliográfica exhaustiva de la especie o en su defecto, el género y/o la familia. En este sentido, el material botánico se identificó en el herbario de la Universidad Tecnológica del Chocó.

Contenido químico de la composición etnofarmacológica. Antes de llevar a cabo el tamizaje fitoquímico a la composición etnofarmacológica, se realizó una revisión de literatura en algunas bases de datos (Current Contens 1998, septiembre de 2009), a fin de identificar estudios de análisis del contenido químico (metabolitos secundarios) y actividades biológicas de las plantas que conforman la composición etnofarmacológica para el tratamiento de la hipertensión. El material vegetal presente en la composición etnofarmacológica se sometió a análisis químico, mediante marcha fitoquímica, se utilizaron los siguientes test de reconocimiento:

- Reacción de cloruro férrico para compuestos fenólicos (CF).

- Reacción de proteínas para taninos (TA).

- Reacción de Shinoda para flavonoides (FL).

- Reacción de Rosenheim para leucoantocianidinas (LE).

Reacción de Kedde para compuestos lactónicos (CA).

Método de la espuma para saponinas (SA).

- Reacción de Lieberman-Burchard para triterpenóides y/o esteroides (TE).

- Reacción de Borntranger para quinonas (QU).

- Reacciones de Mayer, Valser, Reineckato de Amonio y Dragendorff para alcaloides (AL).

Todos estos análisis se realizaron por triplicado (Figura 1):

Determinación de la efectividad del tratamiento médico tradicional aplicado a la cura y/o alivio de pacientes hipertensos. Para avanzar en la validación de los tratamientos médicos tradicionales usados para tratar la hipertensión arterial, se realizó un monitoreo a algunos pacientes declara- 


\section{$25 \mathrm{~g}$ droga seca y pulverizada o extracto}

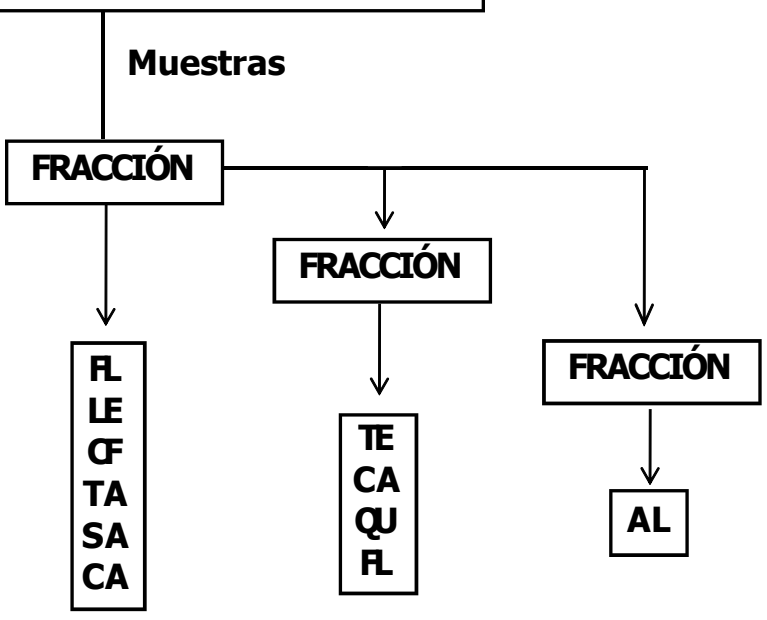

Figura 1. Flujograma resumen de la metodología utilizada para el análisis fitoquímico.

Tabla 1

Plantas medicinales utilizadas en la composición etnofarmacológica para el tratamiento de la hipertensión arterial

\begin{tabular}{clll}
\hline No & Nombres comunes & Nombre científico & Parte utilizada \\
\hline 1 & Guanábana & Annona muricata & Hojas \\
2 & Aguacate & Persea americana Mili & Hojas \\
3 & Mejorana & Majorana hortensis & Toda la planta \\
\hline 4 & Guayaba & Psidium guajava $L$ & Cogollos, hojas \\
5 & Ajo & Allium sativum & Bulbos \\
6 & Borojó & Borojoa patinoi & Frutos \\
\hline
\end{tabular}

dos hipertensos por parte de un médico facultativo. Los pacientes se sometieron al tratamiento médico tradicional propuesto para la cura y/o alivio de la hipertensión arterial, que consistió en el suministro de dos dosis diarias (un vaso aproximadamente) de la composición etnofarmacológica en estudio, de la que se debían tomar un vaso en la mañana y otro antes de acostarse, con recomendaciones como no consumir carne, huevo ni bebidas alcohólicas durante el tiempo que duró el tratamiento (más o menos un mes).

Con la intención de llevar a cabo la documentación de la efectividad y dosificaciones usadas en los tratamientos de los enfermos que padecen de hipertensión, se diseñaron y aplicaron protocolos de entrevistas individuales a quienes se sometieron al tratamiento, acompañado de un monitoreo y seguimiento a través de un expediente clínico, de acuerdo con las pautas para la investigación de la inocuidad y eficacia de los medicamentos herbarios recomendadas por la OMS.

\section{RESULTADOSYDISCUSIÓN}

Identificación y descripción de las plantas medicinales. La composición etnofarmacológica se determinó a una combinación de seis extractos acuosos de plantas medicinales (Tabla 1): extracto acuoso de hojas de guanábana (Annona muricata), extracto acuoso de hojas de aguacate (Persea americana Mili), extracto acuoso de mejorana (Majorana hortensis), extracto acuoso de cogollos de guayaba (Psidium guajava L.), extracto acuoso de ajo (Allium sativum) y extracto acuoso de fruto de borojó (Borojoa patinoi).

La Annona muricata (guanábana), de la familia Annonaceae, es un árbol que alcanza hasta los 10 metros de altura, de follaje compacto, hojas simples, coriáceas verde oscuro, grandes y brillantes; flores bisexuales solitarias o en pares, en tallos cortos que brotan de las ramas viejas; cáliz con tres sépalos diminutos e inconspicuos de color verde; corola 
con seis pétalos de color amarillo. El fruto es una baya colectiva o sin carpo, de forma acorazonada u ovoide, con pericarpio (cáscara) verdoso con tubérculos espiniformes carnosos, la pulpa es blanca y jugosa de sabor agridulce, las semillas de color negro lustroso o castaño (Palomino 2007, citado por Rentería et al. 2009).

El aguacate (Persea americana Mili), de la familia Lauraceae, es un árbol grande o de tamaño mediano, con frecuencia de 20 metros de alto, con una copa muy densa en donde se encuentra un fruto que tiene forma ovalada o esférica.

La mejorana (Origanum majorana) es una hierba perenne de la familia Lamiaceae, cultivada por su uso aromático, que recuerda al pino y a los cítricos; alcanzan un tamaño de unos $60 \mathrm{~cm}$. de altura, algo leñosos en la base, hojas aovadas, enteras, blanquecinas y lanuginosas. Las diminutas flores labiadas surgen reunidas en ramilletes terminales, suelen ser blancas o rosadas. Las semillas son redondas, menudas y rojizas.

La guayaba (Psidium guajava), es una planta perteneciente a la familia Myrtaceae, es un árbol con tronco de corteza suave, delgada, rojo-café, con escamas que caen; hojas verdes, opuestas, elípticas u oblongas, redondeadas en el ápice y base; flores axilares, solitarias, blancas; fruto en baya, aromáticos de diversas formas, según la variedad, carnaza rosada o amarilla.

El ajo (Allium sativum) es una planta perenne de olor fuerte (Lovatis 1994; Cáceres 1995). Puede llegar hasta un metro de altura (Lovatis 1984). Tallo cilíndrico de $50 \mathrm{~cm}$ de altura. Hojas lanceoladas verdes de $30 \mathrm{~cm}$ de largo. Inflorescencia en umbela con flores de pétalos blancos o blancos purpúreos (Cáceres 1995), que por lo general son estériles; fruto en cápsula triangular (Peris et al. 1995). Las flores son de color blanco-rosado (Lovatis 1994), la raíz es un bulbo redondeado constituido por gajos de sabor picante (Lovatis 1994; Cáceres 1995).

El borojó (Borojoa patinoi Cuatr.). Es una especie arbórea perteneciente a la familia Rubiaceae, de $3 \mathrm{~m}$ a $5 \mathrm{~m}$ de altura. Tallo erecto, hojas decusadas, con estípulas bien definidas, coriáceas. Planta dioica. Flores masculinas en capítulos, cáliz corto, prismático o cónico, por lo general actinomorfas, sésiles, pentámeras y a veces tetrámeras desprovistas de ovario o si éste existe, es rudimentario o no funcional. Las flores femeninas son solitarias y terminales con dos pares de estípulas bracteales y seis estigmas más largos; ovario ínfero, con cáliz umbilicado en la base, seis cavidades y muchos óvulos, corola con seis a nueve pétalos, estambres lineales, vacíos o estériles. El fruto es una baya carnosa de 7 cma $12 \mathrm{~cm}$ de largo un diámetro similar, pudiendo ser periforme y por lo general achatado en el ápice, color verde al principio y pardo claro al madurar; pulpa constituida por el mesocarpio y el endocarpio, sin separación aparente con la cáscara.
Contenido químico de la composición etnofarmacológica. La revisión de literatura (Curren Contens 1998, septiembre de 2009) referida a estudios de análisis del contenido químico (metabolítos secundarios) y actividades biológicas de las plantas que conforman la composición etnofarmacológica para el tratamiento de la hipertensión, nos muestra que algunas de estas plantas contienen en su estructura química metabolitos secundarios como: flavonoides, derivados triterpénicos, leucoantocianidinas, saponinas, compuestos fenólicos, antocianinas, alicina, entre otros (Tabla2).

Los análisis fitoquímicos que se realizaron a la composición etnofarmacológica, mostraron resultados de presunción muy positiva para la presencia de compuestos lactónicos como cardiotónicos o terpenlactonas, y altamente positiva de compuestos fenólicos y leucoantocianidinas (Tabla 3).

El resultado de estos análisis fitoquímicos (Tabla 3), junto con información bibliográfica reciente sobre estudios fitoquímicos y farmacológicos de las especies vegetales usadas en la preparación de esta composición etnofarmacológica (Tabla 2), demuestran la presencia de compuestos fenólicos, sobre todo en las hojas y cáscara de guayaba (Psidium guajaba) (Marquina et al. 2008; Chen y Yen 2007); hojas, tallos y flores de mejorana (Majorana ortensis) (Dorman et al. 2004); bulbos de ajo (Allium sativum) (Bozin et al. 2008). Flavonoides: hojas de mejorana (Majorana ortensis) (Lozaya et al. 2002), fruto de borojó (borojoa patinoi) (Mosquera 2005). Compuestos con reconocida actividad biológica antioxidante e hipotensora. Varios estudios han demostrado que la corteza y hojas de guanábana (Annona muricata) tienen actividad hipotensora en ratas (Hasrat et al. 1997, citado por Palomino 2007). Extractos de ajo (Allium sativum) han mostrado efectos antihipertensivos en ratas ( $\mathrm{Al}$ Qattan et al.2003).

Determinación de la efectividad del tratamiento médico tradicional aplicado a la cura y/o alivio de pacientes hipertensos. Para avanzar en la validación de los tratamientos médicos tradicionales empleados para tratar la hipertensión arterial se realizó un monitoreo acompañado de entrevistas a ocho pacientes, todos declarados hipertensos por parte de un médico facultativo, quienes participaron de manera informada y voluntaria en la investigación. Las ocho personas hipertensas que participaron en la investigación, tomaron durante 30 días por las mañanas y por las noches 200 a $250 \mathrm{ml}$ (un vaso aproximadamente) de la composición etnofarmacológica, se tomó la presión arterial a los pacientes antes de iniciar el tratamiento y de nuevo a los 8, 15, 21 y 30 días de continuado el mismo, se registraron resultados de manera progresiva a través de entrevistas, de las que se obtuvo que 98.3\% de los pacientes que se sometieron al tratamiento etnofarmacológico expresaron sentir enorme mejoría en estado de ánimo, eliminando agotamiento, cansancio, fatiga y 
Bioetnia Volumen 7 № 2 (julio-diciembre), 2010






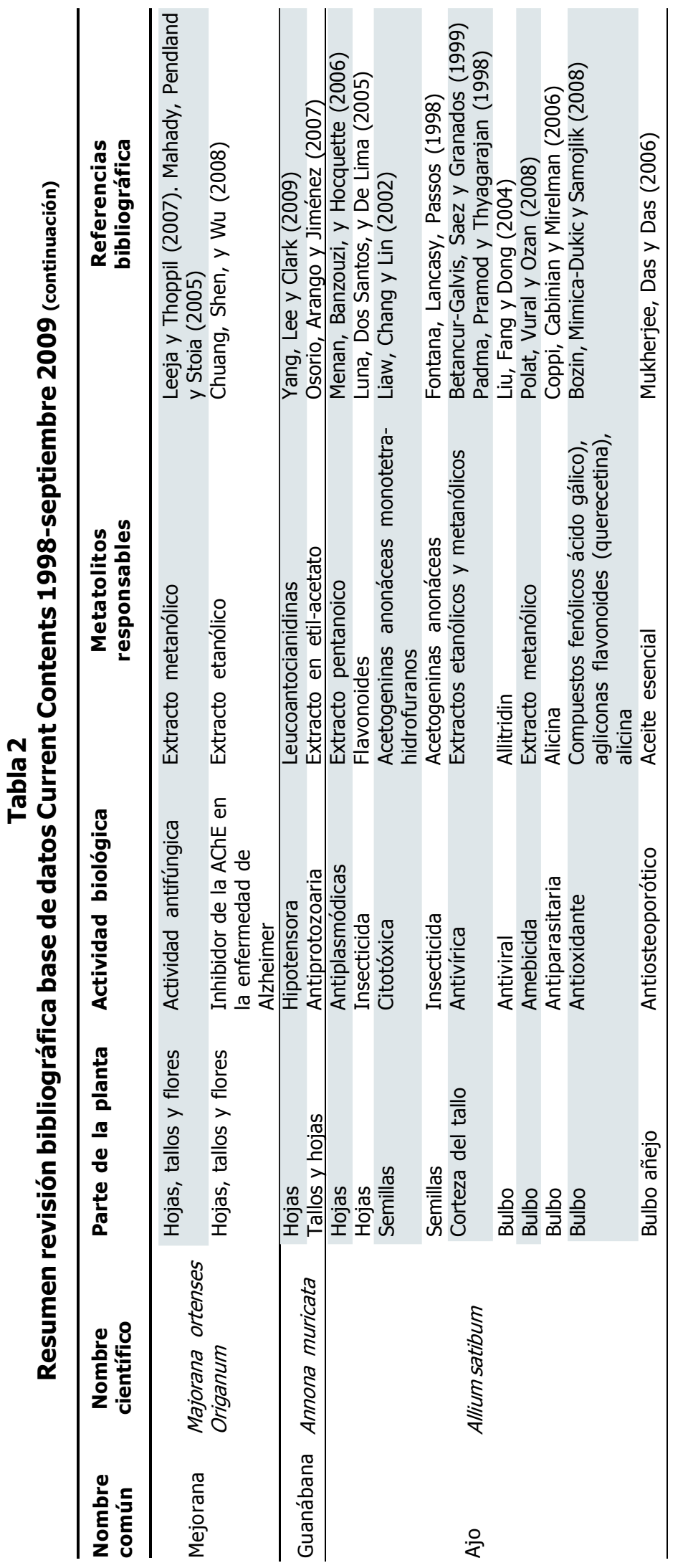

debilidad, inclusive desde la primera semana luego de iniciarse el tratamiento.

La información referida a la medición de la presión arterial de los ocho pacientes, antes, durante y después de la administración de la composición etnofarmacológica se muestra en la Tabla 4.

\section{CONCLUSIONES}

Es posible que los metabolitos secundarios presentes en la composición etnofarmacológica contribuyan al tratamiento de la hipertensión, porque de acuerdo con las actividades biológicas descritas para algunos compuestos fenólicos, se pueden asociar a la prevención de enfermedades inducidas por estrés oxidativo como las enfermedades cardiovasculares. Los posibles efectos protectores informados, se relacionan por lo general con la actividad antioxidante de los mismos.

Las diferentes pruebas con pacientes declarados hipertensos, junto con la presencia de compuestos fenólicos y cardiotónicos en la composición etnofarmacológica, además de los testimonios suministrados por los pacientes, demuestran que esta composición etnofarmacéutica presenta propiedades para curar y/o aliviar la hipertensión arterial.

La efectividad que se obtuvo fue de $98.3 \%$ en relación con los tratamientos y las personas mostraron porcentajes de recuperación de los niveles normales en la presión arterial en un intervalo de 120/80 en promedio, hasta tal punto que abandonaron los tratamientos médicos facultativos para tratar su dolencia (verapamilo, captopril, losartán, etc.).

\section{LITERATURA CITADA}

Abbassy, M. A., Abdelgaleil, S. A. M., Rabie, R. Y. A. 2009. Insecticidal and synergistic effects of Majorana hortensis essential oil and some of its major constituents. Entomol Experimental Applic. 131(3): 225-32.

Betancur-Galvis, L. A., Sáez, J., Granados, H. 1999. Antitumor and antiviral activity of colombian medicinal plant extracts. Memorias do Instituto Oswaldo Cruz. 94 (4): 531-5.

Bozin, B., Mimica-Dukic, N., Samojlik, I. 2008. Phenolics as antioxidants in garlic (Allium sativum L., Alliaceae). Food Chemm. 111 (4): 925-9.

Brandelli, C. L. C., Giordani, R. B., De Carli, G. A. 2005. Indigenous traditional medicine: in vitro anti-giardial activity of plants used in the treatment of diarrhea. Parasitol Res. 104 (6): 1345-9.

Cáceres, A. 1995. Plantas de uso medicinal en Guatemala.. San Carlos: Ed. Universitario, Universidad de San Carlos de Guatemala; p. 63-6.

Castro, O., Gutiérrez, J. M., Barrios, M. 1999. Neutralization of the haemorrhagic effect induced by Bothrops asper (Serpentes: Viperidae) venom with tropical plant extracts. Rev Biol Trop. 47(3): 605-15

Chen, K. C., Hsieh, C. L., Peng, C. C. 2008. Brain derived metastatic 
Tabla 3

Resultados marcha analítica de la composición etnofarmacológica para tratar HTA

\begin{tabular}{lccc}
\hline Prueba & $\begin{array}{c}\text { Extracto acuoso } \\
\text { (Fracción A) }\end{array}$ & $\begin{array}{c}\text { Solución orgánica } \\
\text { (Fracción B) }\end{array}$ & $\begin{array}{c}\text { Solución acuosa ácida } \\
\text { (Fracción C) }\end{array}$ \\
\hline Compuestos fenólicos & +++ & \\
Cumarinas & - & - \\
Leucoantocianidinas & +++ & ++ \\
Saponinas & - & - \\
Taninos & - & - \\
Flavonoides & - & \\
Compuestos lactónicos & - & - \\
Triterpenos y/o esteroides & & \\
Quinonas & & \\
Alcaloides & & \\
\hline
\end{tabular}

Negativo (-), Positivo (+), Muy positivo (++), Altamente positivo (+++), Dudoso (+/-)

Tabla 4

Mediciones de presión de pacientes sometidos al tratamiento

\begin{tabular}{|c|c|c|c|c|}
\hline \multirow[t]{2}{*}{ Pacientes } & \multirow[t]{2}{*}{ Edad } & \multirow[t]{2}{*}{ Sexo } & \multicolumn{2}{|c|}{ Mediciones promedios de presión arterial $(\mathbf{m m ~} \mathbf{H g})$} \\
\hline & & & Antes del tratamiento & Después del tratamiento \\
\hline P1 & 53 & M & $180-70$ & $110-90$ \\
\hline $\mathrm{P} 2$ & 52 & M & $190-80$ & $120-70$ \\
\hline P3 & 59 & $\mathrm{~F}$ & $160-80$ & $120-80$ \\
\hline P4 & 55 & $\mathrm{~F}$ & $160-80$ & $120-80$ \\
\hline P5 & 46 & $\mathrm{~F}$ & $180-90$ & $120-90$ \\
\hline P6 & 48 & M & $180-70$ & $100-80$ \\
\hline P7 & 40 & M & $160-80$ & $120-80$ \\
\hline P8 & 51 & M & $180-80$ & $120-70$ \\
\hline
\end{tabular}

prostate cancer DU-145 cells are effectively inhibited in vitro by guava (Psidium gujava L.) leaf extracts. Nutr Cancer. 58 (1): 93-106.

Cheng, F. C., Shen, S. C., Wu, J. S. B. 2009. Effect of guava (Psidium guajava

L.) Leaf extract on glucose uptake in rat hepatocytes. J Food Sci. 74 (5): H132-H8.

Chen, H. Y., Yen, G. C. 2007. Antioxidant activity and free radical-scavenging capacity of extracts from guava (Psidium guajava L.) leaves Source. Food Chemistry. 101(2): 686-94.

Choi, S. Y., Hwang, J. H., Park, S. Y. 2008. Fermented guava leaf extract inhibits LPS-induced COX-2 and iNOS expression in mouse macrophage cells by inhibition of transcription factor NFkappa B. Phytother Res. 22 (8): 10304.

Chuang, P. T., Shen, S. C., Wu, N. J. 2008. Anti-peroxidation effect of guava (Psidium guajava Linn.) leaf soluble solids in vitro and in streptozotocin/ nicotinamide-induced diabetic rats. J Sci Food Agricult. 88 (12): 21739.

Coppi, A., Cabinian, M., Mirelman, D. 2006. Antimalarial activity of allicin, a biologically active compound from garlic cloves. Antimicrobiol Agents
Chemother. 50 (5): 1731-7.

De Almeida, A. P., Miranda, M. M. F. S., Simoni, I. C. 1998. Flavonol monoglycosides isolated from the antiviral fractions of Persea americana (Lauraceae) leaf infusión. Phytother Res. 12 (8): 562-7.

Domergue, F., Helms, G. L., Prusky, D. 2000. Antifungal compounds from idioblast cells isolated from avocado fruits. Phytochemistry. 54 (2): 1839.

Dorman, H. J. D., Bachmayer, O., Kosar, M. 2004. Antioxidant properties of aqueous extracts from selected Lamiaceae species grown in Turkey. $J$ Agricult Food Chem. 52 (4): 762-70.

Fontana, J.D., Lancasy, F.M., Passos, M. 1998. Selective polarity and adsorption-guided extraction purification of Annona sp. polar acetogenins and biological assay against agricultural pests. Applied Biochem Biotechnol. 70 (2): 67-76.

Hoque, M. D. M., Bari, M. L., Inatsu, Y., et al. 2007. Antibacterial activity of guava (Psidium guajava L.) and neem (Azadirachta indica A. Juss.) extracts against foodborne pathogens and spoilage bacteria. Foodborne Path Dis. 4 (4): 481-8. 


\section{Efectividad de la etnofarmacología en hipertensión arterial. CA Rentería}

Jaiarj, P., Khoohaswan, P., Wongkrajang, Y. 1999. Anticough and antimicrobial activities of Psidium guajava Linn. Leaf extract. JEthnopharmacol. 67 (2): 203-12.

Kaileh, M., Vanden Berghe, W., Boone, E. 2007. Screening of indigenous Palestinian medicinal plants for potential anti-inflammatory and cytotoxic activity. J Ethnopharmacol. 113 (3): 510-6.

Kawagishi, H., Fukumoto, Y., Hatakeyama, M. 2001. Liver injury suppressing compounds from avocado (Persea americana). J Agricult Food Chem. 49 (5): 2215-21.

Kawakami, Y., Nakamura, T., Hosokawa, T. 2009. Antiproliferative activity of guava leaf extract via inhibition of prostaglandin endoperoxide $\mathrm{H}$ synthase isoforms. Prostagland Leukotr Essent Fatty Acids. 80 (5-6): 239-45.

Koua, K.H., Han, S.H. 1998. Evaluation of the larvicide effect of an aqueous extract of Persea americana (Miller, 1768), Lauraceae, on Anopheles gambiae (Giles, 1902), Diptera Culicidae. Inverteb Reproduct Develop. 34 (1): $97-100$

Lamiri, A., Lhaloui, S., Benjilali, B. 2001. Insecticidal effects of essential oils against Hessian fly, Mayetiola destructor (Say). Field Crops Res. 71 (1): 9-15.

Leeja, L., Thoppil, J. E. 2007. Antimicrobial activity of methanol extract of Origanum majorana L. (Sweet marjoram). J Environm Biol. 28 (1): 1456.

Liaw, C. C., Chang, F. R., Lin, C. Y. 2002. New cytotoxic monotetrahydrofuran annonaceous acetogenins from Annona muricata. JNat Produc. 65 (4): 4705.

Lin, J., Puckree, T., Mvelase, T. P. 2002. Anti-diarrhoeal evaluation of some medicinal plants used by Zulu traditional healers. J Ethnopharmacol. 79 (1): 53-6.

Liu, Z. F., Fang, F., Dong, Y. S. 2004. Experimental study on the prevention and treatment of murine cytomegalovirus hepatitis by using allitridin. Antiviral Res. 61 (2): 125-8.

Lovatis, S., Castellani, F. 1994. Alimentos y plantas medicinales. Bogotá, DC: Ed. Norma SA; p. 118-9.

Lozoya, X., Reyes-Morales, H., Chávez-Soto, M. A. 2002. Intestinal antispasmodic effect of a phytodrug of Psidium guajava folia in the treatment of acute diarrheic disease. J Ethnopharmacol. 83 (1-2): 19-24.

Luna, J. D., dos Santos, A. F., de Lima, M. R. F. 2005. A study of the larvicidal and molluscicidal activities of some medicinal plants from northeast Brazil. J Ethnopharmacol. 97 (2): 199-206.

Mahady, G. B., Pendland, S. L., Stoia, A. 2005. in vitro susceptibility of Helicobacter pylori to botanical extracts used traditionally for the treatment of gastrointestinal disorders. Phytother Res. 19 (11): 988-91.

Marquina, V., Araújo, L., Ruiz, J. 2008. Composicion quimica y capacidad antioxidante en fruta, pulpa y mermelada de guayaba (Psidium guajava L.) Arch Latinoam Nutr. 58 (1): 98-102.

Menan, H., Banzouzi, J. T., Hocquette, A. 2006. Antiplasmodial activity and cytotoxicity of plants used in West African traditional medicine for the treatment of malaria. J Ethnopharmacol. 105 (1-2): 131-6.

Miranda, M. M. F. S., Almeida, A. P., Costa, S. S. 1997. in vitro activity of extracts of Persea americana leaves on acyclovirresistant and phosphonoacetic resistant herpes simplex virus. Phytomedicine. 4 (4): 347-52.

Mosquera, L. H., Ríos Hurtado, A., Zapata Porras, S. 2005. Obtención de una materia prima con valor agregado mediante secado por aspersión, a partir del fruto fresco de borojó (Borojoa patinoi Cuatr.). Revista Institucional Universidad Tecnológica del Chocó D.L.C. 23: 5-10.

Mukherjee, M., Das, A. S., Das, D. 2006. Role of oil extract of garlic (Allium sativum Linn.) on intestinal transference of calcium and its possible correlation with preservation of skeletal health in an ovariectomized rat model of osteoporosis. Phytother Res. 20 (5): 408-15.
Nair, R., Chanda, S. 2007. in vitro antimicrobial activity of Psidium guajava L. leaf extracts against clinically important pathogenic microbial strains. Braz J Microbiol. 38 (3): 452-8.

Ojewole, J. A. O. 2006. Antiinflammatory and analgesic effects of Psidium guajava Linn. (Myrtaceae) leaf aqueous extract in rats and mice. Meth Find Experim Clin Pharmacol. 28 (7): 441-6.

Ojewole, J. A. O., Amabeoku, G. J. 2006. Anticonvulsant effect of Persea americana Mill (Lauraceae) (avocado) leaf aqueous extract in mice. Phytother Res. 20 (8): 696-700.

Osorio, E., Arango, G. J., Jiménez, N. 2007. Antiprotozoal and cytotoxic activities in vitro of Colombian Annonaceae. J Ethnopharmacol. 111 (3): 630-5.

Pachanawan, A., Phumkhachorn, P. R., Rathanachaikunsopon, P. 2008. Potential of Psidium guajava suplemented fisch diets in controlling aeromonas hydrophila infection in tilapia (Oreochromis niloticus). J Biosci Bioengin. 106 (5): 419-24.

Padma, P., Pramod, N. P., Thyagarajan, S. P. 1998. Effect of the extract of Annona muricata and Petunia nyctaginiflora on herpes simplex virus. $J$ Ethnopharmacol. 61 (1): 81-3.

Palomino F. C. 2007. Efecto del extracto etanólico de hojas de Annona muricata L. (guanábana) sobre la hiperglicemia inducida con aloxano en ratas. Tesis de grado para optar el título de Magíster en Farmacología con mención en Farmacología Experimental. Tegucigalpa, Universidad Nacional Mayor de San Marcos.

Pelegrini, P. B., Murad, A. M., Silva, L. P. 2008. Identification of a novel storege glycine-rich peptide from guava (Psidium guajava) sedes with activity against Gram-negative bacteria. Peptides. 29 (8): 1271-9.

Peris J. B., Stubing, G., Vanaclocha, B. 1995. Fitoterapia aplicada. Valencia: Colegio Oficial de Farmacéuticos de Valencia; 628 pp.

Polat, Z. A., Vural, A., Ozan, F. 2008. in vitro evaluation of the amoebicidal activity of garlic (Allium sativum) extract on Acanthamoeba castellanii and its cytotoxic potential on corneal cells. J Ocular Pharmacol Therap. 24 (1): 8-14.

Rai, P.K., Jaiswal, D., Mehta, S. 2009. Anti-hyperglycaemic potential of Psidium guajava raw fruit peel. Indian J Med Res. 129 (5): 561-5.

Rodríguez, J., Ruiz, F., Peñaloza, E., Eslava , J., Gómez, L. C., Sánchez, H., et al. 2009. Encuesta Nacional de Salud 2007. Resultados Nacionales. Bogotá, DC: Ministerio de la Protección Social, COLCIENCIAS, SEI S.A., Pontificia Javeriana Universidad, CENDEX; 343 pp.

Santos, F. A., Rao, V. S. N., Silveira, E. R. 1998. Investigations on the antinociceptive effect of Psidium guajava leaf essential oil and its major constituents. Phytother Res. 12 (1): 24-7.

Seo, N., Ito, T., Wang, N. L. 2005. Anti-allergic Psidium guajava extracts exert an antitumor effect by inhibition of $\mathrm{T}$ regulatory cells and resultant augmentation of Th1 cells. Anticancer Res. 25 (6A): 3763-70.

Yamanaka, F., Hatano, T., Ito, H. 2008. Antibacterial effects of guava tannins and related polyphenols on vibrio and aeromonas species. Nat Product Comm. 3 (5): 711-20.

Yang, Y. C., Lee, S. H., Clark, J. M. 2009. Ovicidal and adulticidal activities of Origanum majorana essential oil constituents against insecticidesusceptible and pyrethroid/malathion-resistant Pediculus humanus capitis (Anoplura: Pediculidae). J Agricult Food Chem. 57 (6): 2282-7.

Yazdanparast, R., Shahriyary, L. 2008. Comparative effects of Artemisia dracunculus, Satureja hortensis and Origanum majorana on inhibition of blood platelet adhesion, aggregation and secretion. Vasc Pharmacol. 48 (1): $32-7$.

Zahir, A. A., Rahuman, A. A., Kamaraj, C. 2009. Laboratory determination of efficacy of indigenous plant extracts for parasites control. Parasitol Res: 105 (2): 453-61. 